\title{
A Support Vector Machine based method to distinguish proteobacterial proteins from eukaryotic plant proteins
}

\author{
Ruchi Verma, Ulrich Melcher \\ From Proceedings of the Ninth Annual MCBIOS Conference. Dealing with the Omics Data Deluge \\ Oxford, MS, USA. 17-18 February 2012
}

\begin{abstract}
Background: Members of the phylum Proteobacteria are most prominent among bacteria causing plant diseases that result in a diminution of the quantity and quality of food produced by agriculture. To ameliorate these losses, there is a need to identify infections in early stages. Recent developments in next generation nucleic acid sequencing and mass spectrometry open the door to screening plants by the sequences of their macromolecules. Such an approach requires the ability to recognize the organismal origin of unknown DNA or peptide fragments. There are many ways to approach this problem but none have emerged as the best protocol. Here we attempt a systematic way to determine organismal origins of peptides by using a machine learning algorithm. The algorithm that we implement is a Support Vector Machine (SVM).
\end{abstract}

Result: The amino acid compositions of proteobacterial proteins were found to be different from those of plant proteins. We developed an SVM model based on amino acid and dipeptide compositions to distinguish between a proteobacterial protein and a plant protein. The amino acid composition (AAC) based SVM model had an accuracy of $92.44 \%$ with 0.85 Matthews correlation coefficient (MCC) while the dipeptide composition (DC) based SVM model had a maximum accuracy of $94.67 \%$ and 0.89 MCC. We also developed SVM models based on a hybrid approach (AAC and DC), which gave a maximum accuracy $94.86 \%$ and a 0.90 MCC. The models were tested on unseen or untrained datasets to assess their validity.

Conclusion: The results indicate that the SVM based on the AAC and DC hybrid approach can be used to distinguish proteobacterial from plant protein sequences.

\section{Background}

Bacterial plant pathogens are a major threat to global food security [1]. Half of the bacterial species causing major food losses in the world belong to the major phylum Proteobacteria (Figure 1). They are found predominantly in the class Gammaproteobacteria (Xanthomonas, Pseudomonas and Erwinia) and also in the class Betaproteobacteria (Ralstonia). Gammaproteobacteria include in addition a wide variety of several medically, ecologically and scientifically important groups such as Enterobacteriaceae (Escherichia coli), Vibrionaceae and

\footnotetext{
* Correspondence: ulrich.melcher@okstate.edu

* Correspondence: ulrich.melcher@okstate.edu University, Stillwater, OK 74078 USA
}

Pseudomonadaceae. Also, beneficial bacteria, such as nitrogen fixing, ammonia oxidizing and iron fixing bacteria are members of this phylum. Betaproteobacteria also include ammonia oxidizing and arsenic resistant bacteria with Burkholderiales as one of the major classes. Alphaproteobacteria is dominated mostly by nitrogen-fixing bacteria and agrobacteria. Deltaproteobacteria and Epsilonproteobacteria have aerobic genera and curved to spirilloid Wolinella spp., respectively. Zetaproteobacteria is composed of a sole member: Mariprofundus ferrooxydans which oxidizes ferrous to ferric iron [2].

Several methods are being developed to detect phytopathogens involving macromolecular sequencing, 


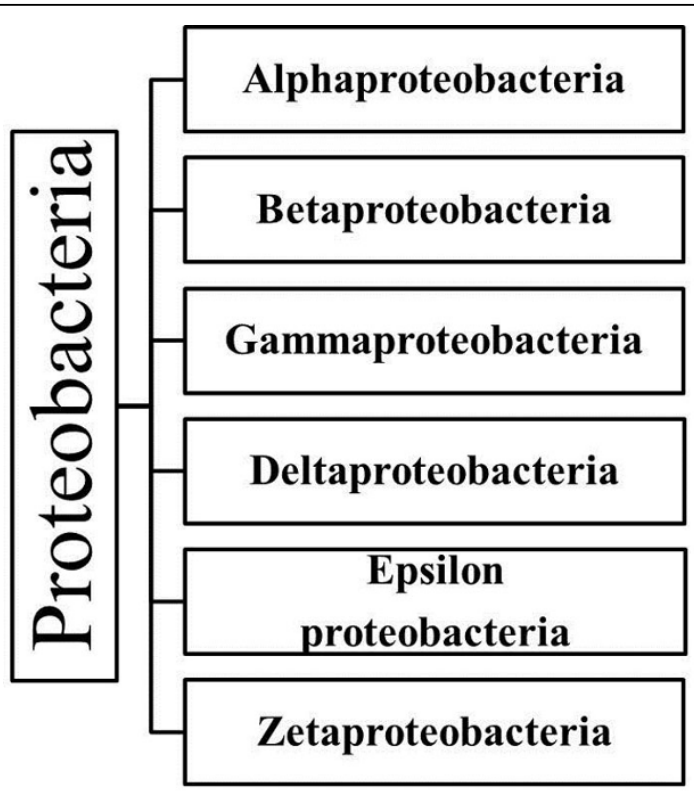

It has a sole member, Mariprofundus ferrooxydans

According to Bergey's manual of bacteriology Agrobacterium, marine

Burkholderiales, ammonia oxidizing, arsenic resistant soil bacteria

\section{Pseudomonas, Xanthomonas,} Acidithiobacillus

Ferric iron-reducing, sulfur reducing

Inhabit the digestive tract of animals and serve as symbionts or pathogens

\section{Nitrogen-fixing (Rhizobium),}

Figure 1 The subgroups of proteobacteria and the main members of each subgroup

especially nucleotide sequencing [3,4]. With the advent of next generation sequencing, testing of diseased or quarantined plants for the presence of proteobacteria will rely increasingly on massive DNA sequencing. Peptide mass spectroscopy also shows promise in such screening. The analysis of nucleotide sequences typically involves assembly of sequence reads into contigs followed by analysis using Blast [5] search to identify pathogen-derived contigs. This approach is limited in that it only identifies potential pathogens whose nucleotide sequences are included in the searched database. Thus, there is a strong need for methods to find the organismal origin of unknown DNA or peptide fragments to identify potential pathogen sequences. Machine learning techniques, such as support vector machines (SVMs) and neural networks have been used successfully to develop classifiers for a number of different biolgocial problems including predicting different categories of proteins [6-14]. As a first step towards detecting pathogenic bacteria spp., we evaluated whether a machine learning algorithm, SVM, could distinguish between proteobacterial (potential pathogen) and plant (host) proteins. Thus, we assembled datasets of proteobacterial and plant host proteins for this study. We focused on amino acid, rather than nucleotide residues, because of the greater variety of residues that can be present at any one position, allowing subtle evolutionary forces to play a role in shaping the protein sequence and its properties. 
Table 1 Total number of pathogen proteins taken from Uniprot and number of proteins remaining after redundancy filtering at 3 different percentages.

\begin{tabular}{|c|c|c|c|c|}
\hline Positive dataset (Pathogen) & $\begin{array}{c}\text { Total number of proteins } \\
\text { (reviewed) }\end{array}$ & $\begin{array}{l}90 \% \\
\text { redundancy }\end{array}$ & $\begin{array}{l}50 \% \\
\text { redundancy }\end{array}$ & $\begin{array}{l}30 \% \\
\text { redundancy }\end{array}$ \\
\hline $\begin{array}{l}\text { Agrobacterium tumefaciens (Rhizobium } \\
\text { radiobacter) }\end{array}$ & 104 & 103 & 103 & 103 \\
\hline Burkholderia phymatum & 333 & 333 & 333 & 333 \\
\hline Pseudomonas aeruginosa (ATCC) & 1217 & 1216 & 1211 & 1211 \\
\hline Xanthomonas oryzae pv. Oryzae & 411 & 410 & 410 & 410 \\
\hline Ralstonia solanacearum & 601 & 601 & 599 & 599 \\
\hline Rhizobium etli (ATCC) & 424 & 421 & 421 & 421 \\
\hline Rhizobium meliloti & 48 & 47 & 47 & 47 \\
\hline Methylobacterium nodulans & 213 & 213 & 213 & 213 \\
\hline Desulfobacterales autotrophicum (ATCC) & 157 & 157 & 157 & 157 \\
\hline Total & 3508 & 3501 & 3494 & 3494 \\
\hline Total after blastclust on cumulative data & - & 3408 & 3230 & 3203 \\
\hline
\end{tabular}

best-trained models, we tested the models on unseen/ blind or untrained data not used for training the SVM. From Uniprot we downloaded non-redundant proteins for three species of proteobacteria (Serratia marcesens, Acidovorax citrulli, Rhizobium fredii) and three plant species (Solanum lycopersicum, Phaseolus vulgaris, Cucurbita pepo).

\section{Feature Vectors used}

Amino Acid Composition (AAC): Each protein was represented as a vector of 20 features, each corresponding to the fractional composition of an amino acid. This set of feature vectors was presented as input to SVM. Separate amino acid frequencies were calculated for both sets of proteins (proteobacteria and plants). The $\mathrm{AAC}$ was calculated by the following equation:

$$
\text { Fraction of amino acid } \mathrm{x}=\frac{\text { Total number of amino acid } x}{\text { Total number of amino acids in protein }}
$$

where $\mathrm{x}$ can be any amino acid residue.
Dipeptide Composition (DC): Each protein was represented as a vector of 400 features for the $20 \times 20$ possible combinations of amino acids. The DC was calculated by the following equation:

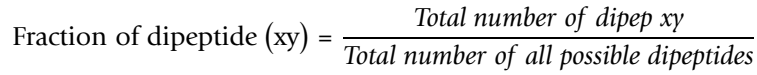

where dipeptide (xy) is one of 400 possible dipeptides.

Hybrid (AAC+DC): The AAC and DC feature vectors were merged to yield feature vectors of 420 features (20 +400 ).

\section{Support Vector Machine}

An SVM is a kernel-based margin classifier, which uses both statistics and optimization. It draws an optimal hyper-plane in a high dimensional feature space that defines a boundary that maximizes the margin between data samples in two classes, therefore giving a better generalization property (Figure 3). Specifically, $\mathrm{SVM}^{\text {light }}$, which is an implementation (in $\mathrm{C}$ language)

Table 2 Total number of plant proteins taken from Uniprot and number of proteins remaining after redundancy filtering at 3 different percentages.

\begin{tabular}{|c|c|c|c|c|}
\hline Negative dataset (Plant host) & Total number of proteins (reviewed) & $90 \%$ redundancy & $50 \%$ redundancy & $30 \%$ redundancy \\
\hline Triticum aestivum & 357 & 315 & 292 & 291 \\
\hline Oryza sativa & 87 & 86 & 86 & 86 \\
\hline Solanum tuberosum & 390 & 314 & 308 & 308 \\
\hline Arabidopsis thaliana & 1000 & 968 & 857 & 852 \\
\hline Cucurbita maxima & 26 & 25 & 25 & 25 \\
\hline Citrus sinensis & 93 & 91 & 91 & 91 \\
\hline Vitis vinefera & 161 & 154 & 152 & 152 \\
\hline Hordeum vulgare & 348 & 323 & 307 & 307 \\
\hline Pisum sativum & 371 & 347 & 335 & 334 \\
\hline Glycine max & 373 & 346 & 324 & 324 \\
\hline Total & 3206 & 2969 & 2777 & 2770 \\
\hline Total after blastclust on cumulative data & - & 2631 & 2284 & 2277 \\
\hline
\end{tabular}



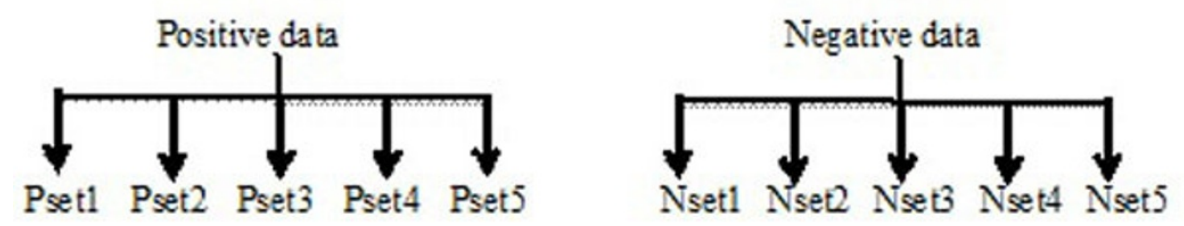

\begin{tabular}{|c|c|c|c|}
\hline \multicolumn{2}{|c|}{ Training set } & \multirow[b]{2}{*}{ Pset5 Nset5 } & Testing set \\
\hline Set1 & $\begin{array}{l}\text { Pset1 Nset1 } \\
\text { Pset2 Nset2 } \\
\text { Pset3 Nset3 } \\
\text { Pset4 Nset4 }\end{array}$ & & Set1 \\
\hline Set2 & $\begin{array}{l}\text { Pset1 Nset1 } \\
\text { Pset2 Nset2 } \\
\text { Pset3 Nset3 } \\
\text { Pset } 5 \text { Nset } 5\end{array}$ & Pset4 Nset4 & Set2 \\
\hline Set3 & $\begin{array}{l}\text { Pset1 Nset1 } \\
\text { Pset2 Nset2 } \\
\text { Pset4 Nset4 } \\
\text { Pset5 Nset5 }\end{array}$ & Pset3 Nset3 & Set3 \\
\hline Set4 & $\begin{array}{l}\text { Pset1 Nset1 } \\
\text { Pset3 Nset3 } \\
\text { Pset4 Nset } 4 \\
\text { Pset5 Nset } 5\end{array}$ & Pset2 Nset2 & Set4 \\
\hline Set5 & $\begin{array}{l}\text { Pset2 Nset2 } \\
\text { Pset3 Nset3 } \\
\text { Pset4 Nset4 } \\
\text { Pset5 Nset } 5\end{array}$ & Pset1 Nset1 & Set5 \\
\hline
\end{tabular}

Figure 2 Construction of datasets using five-fold cross validation. Pset is for positive dataset (proteobacteria) and Nset is for negative dataset (plants)

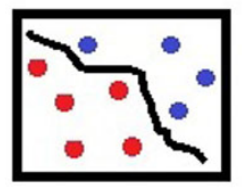

Input space

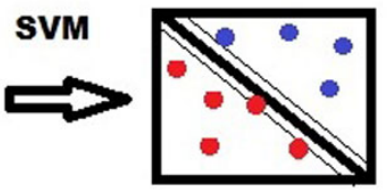

Output feature space

Figure 3 The concept of Support Vector Machine (SVM) in feature differentiation.

of SVM, has been used in this study. The SVM ${ }^{\text {light }}$ package can be downloaded from http://www.joachims. org for non-commercial or academic use [16]. In this study we used the SVM concept for the classification of proteobacteria and plant (host) proteins. Learning was carried out by using three kinds of kernels: the linear $(t=0)$, the polynomial $(t=1)$ and the Radial Basis Function (RBF) $(t=2)$. We obtained the best performance from the RBF. 


\section{Evaluation}

Evaluation of the performance of the three models is threshold dependent. The performance of our method was computed by using the following standard parameters $[17,18]$.

(a) Sensitivity or coverage of positive examples: percent of proteobacterial proteins correctly predicted

Sensitivity $(\mathrm{Sn})=\frac{\mathrm{TP}}{\mathrm{TP}+\mathrm{FN}} \times 100$

(b) Specificity or coverage of negative examples: percent of plant proteins correctly predicted as plant protein

Specificity $(\mathrm{Sp})=\frac{\mathrm{TN}}{\mathrm{TN}+\mathrm{FP}} \times 100$

(c) Accuracy: percent of correctly predicted proteins (bacterial and plant proteins).

Accuracy $($ Acc $)=\frac{\mathrm{TP}+\mathrm{TN}}{\mathrm{TP}+\mathrm{FN}+\mathrm{FP}+\mathrm{FN}} \times 100$

(d) Matthews correlation coefficient (MCC) is considered to be the most robust parameter of any class prediction method [19]. MCC equal to 1 is regarded as perfect prediction while 0 suggests completely random prediction.

$$
\mathrm{MCC}=\frac{(\mathrm{TP} \times \mathrm{TN})-(\mathrm{FP} \times \mathrm{FN})}{\sqrt{(\mathrm{TP}+\mathrm{FP})(\mathrm{TP}+\mathrm{FN})(\mathrm{TN}+\mathrm{FP})(\mathrm{TN}+\mathrm{FN})}}
$$

where TP represents truly predicted proteobacterial proteins, and TN represents truly predicted plant proteins. FP and FN are falsely predicted proteobacterial and plant proteins, respectively.

\section{Results and discussion}

To test whether the AAC of proteobacterial and plant proteins differ significantly, we calculated AAC for both the proteobacterial (Table 1) and plant (Table 2) proteins datasets (Figure 4). We observe differences of AAC between proteobacteria and plants with respect to alanine, cysteine, glycine, lysine, arginine and serine. We also calculated the DC for these two datasets (figure not shown). We input the following vector sets for the SVM: AAC, DC and a hybrid of AAC and DC [20] models. We trained all three kernels (linear (Table 3), polynomial (Table 4) and RBF (Table 5) to identify the best-trained kernel. Comparison of the accuracies and MCCs obtained by all three kernels revealed that the RBF kernel performed best with all three redundancy percentages (Table 5). At $90 \%$ redundancy the SVM achieved a maximum accuracy of $92.44 \%$ and a 0.85 MCC for the AAC model [RBF parameters: $\mathrm{g}=0.04, \mathrm{c}$ $=4, \mathrm{j}=1]$, a maximum accuracy of $94.67 \%$ and 0.89 MCC for the DC model $[g=0.02, c=6, j=2]$ and for the hybrid model a maximum accuracy of $94.86 \%$ and a

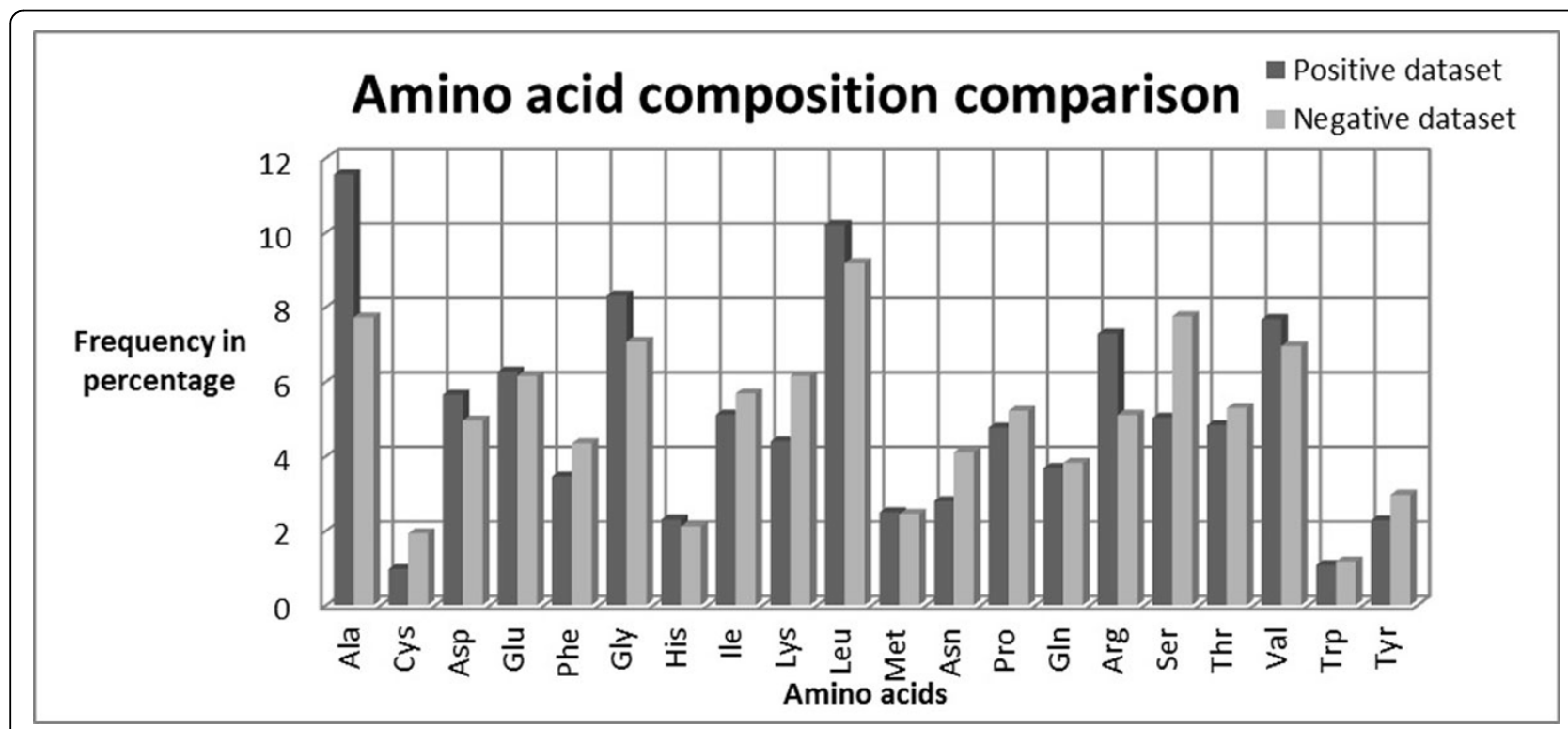

Figure 4 Comparative amino acid compositions of positive dataset (proteobacteria) and negative dataset (plants). 
Table 3 Results of SVM models based on AAC, DC and hybrid (AAC+DC) features at three different redundancy percentages using the linear kernel $(t=0)$.

\begin{tabular}{|c|c|c|c|c|c|c|}
\hline \multirow[t]{2}{*}{ Redundancy(percentage) } & \multicolumn{2}{|c|}{ Amino acid composition (AAC) } & \multicolumn{2}{|c|}{ Dipeptide composition (DC) } & \multicolumn{2}{|c|}{ Hybrid $(A A C+D C)$} \\
\hline & Accuracy (\%) & MCC & Accuracy (\%) & MCC & Accuracy (\%) & MCC \\
\hline 30 & 87.87 & 0.75 & 90.74 & 0.81 & 89.10 & 0.78 \\
\hline 50 & 87.45 & 0.74 & 90.87 & 0.81 & 91.81 & 0.83 \\
\hline 90 & 87.45 & 0.74 & 90.87 & 0.81 & 89.33 & 0.78 \\
\hline
\end{tabular}

$0.90 \mathrm{MCC}[\mathrm{g}=0.01, \mathrm{c}=8, \mathrm{j}=1]$. At $50 \%$ redundancy, maximum accuracies for the AAC, DC and hybrid models were $91.62 \%(\mathrm{MCC} 0.83)$ [ $g=0.04, \mathrm{c}=2, \mathrm{j}=1]$, 94.12\% (MCC 0.88) $[\mathrm{g}=0.02, \mathrm{c}=4, \mathrm{j}=1]$ and $94.49 \%$ (MCC 0.89) [ $\mathrm{g}=0.01, \mathrm{c}=8, \mathrm{j}=2$ ] respectively. At $30 \%$ redundancy, the maximum accuracies of the AAC, DC and hybrid models were $92.30 \%(\mathrm{MCC} 0.84)$ [ $\mathrm{g}=0.05$, c $=1, \mathrm{j}=1]$, 93.72\% (MCC 0.87) $[\mathrm{g}=0.03, \mathrm{c}=2, \mathrm{j}=2]$ and $93.84 \%$ (MCC 0.88) $[\mathrm{g}=0.01, \mathrm{c}=4, \mathrm{j}=2]$. As shown in Table 5 we achieved maximum accuracy with the hybrid model at $90 \%$ redundancy.

The result of the validation datatsets on six species (on all three models) are shown in Table 6. The hybrid model trained at $90 \%$ redundancy had the best accuracy only with exception of Rhizobium fredii for which the $50 \%$ redundant model was better. As can be seen from Table 5, the hybrid model at $90 \%$ redundancy performed best overall for most species. It is possible that the decrease in performance obtained by removing more proteins based on their similarities is not due to the identity value, but due to a resulting imbalance in the training datasets since the redundancy criteria affected proteobacterial protein numbers more strongly than they did the plant protein numbers. Because these estimates are sensitive to the threshold for distinguishing positives from negatives, we constructed a ROC curve to examine the model's accuracy. ROC has been used to show the accuracy of constructed models [21-29]. The ROC curve is a graphical representation of sensitivity (true positive rate) vs. one minus specificity (false positive rate or true negative rate) for any binary classifier system [30]. It is a threshold independent evaluation parameter and gives a value known as Area Under Curve (AUC) (Figure 5) which shows the performance of a classifier in a two class problem [31]. The higher the AUC, the more accurate the model. In the present study the AUC for hybrid model was 0.985 and therefore demonstrated the accuracy of the model.

This SVM model can be used to assign a query sequence as to whether it originated from a plant or proteobacterium, thus enabling timely detection of the infection. It may also be used to identify food contamination with bacteria by screening samples by sequencing. SVM models can be used to work in the area of animal proteins. As we have developed a model for plant and proteobacteria, another model can be designed for animal protein and pathogenic proteobacterial proteins. Thus, SVMs can be used in a variety of fields of study.

\section{Conclusion}

The SVM models based on the hybrid approach using both amino acid and dipeptide features exhibited the maximum accuracy on both threshold dependent and threshold independent parameters. Best results were obtained with an RBF kernel and considering protein sets that did not contain any proteins that are more than $90 \%$ identical to another protein in the dataset. SVMs have great potential to handle large datasets and thus can be used for sorting proteobacterial sequences from a mixed background, like those found in metagenomic sequence data. As such, an SVM classifier would be a step forward in surveillance techniques for bacteria that lack previously characterized relatives. It may be useful for determining protein sequences obtained from non-sequenced genomes not yet present in Genbank. Other features like domains specific to nitrogen oxidising or fixing bacteria can also be used even to distinguish a pathogenic proteobacterium from a nonpathogenic proteobacterium. This may be used to

Table 4 Results of SVM models based on AAC, DC and hybrid (AAC+DC) features dataset at three different redundancy percentages using polynomial kernel $(t=1)[d$ is another parameter used in this kernel and its value is given in parentheses].

\begin{tabular}{ccccccc}
\hline Redundancy(percentage) & \multicolumn{2}{c}{ Amino acid composition (AAC) } & \multicolumn{2}{c}{ Dipeptide composition (DC) } & \multicolumn{2}{c}{ Hybrid (AAC+DC) } \\
\hline & Accuracy (\%) & MCC & Accuracy (\%) & MCC & Accuracy (\%) & MCC \\
\hline 30 & (d5) 89.63 & 0.78 & (d2) 92.06 & 0.84 & (d6) 91.29 & 0.82 \\
50 & (d4) 88.88 & 0.77 & (d2) 91.98 & 0.83 & $(d 4) 90.68$ & 0.81 \\
90 & (d5) 89.46 & 0.78 & (d2) 92.54 & 0.85 & (d4) 91.26 & 0.82 \\
\hline
\end{tabular}


Table 5 Results of SVM models based on AAC, DC and hybrid (AAC+DC) features at three different redundancy percentages using RBF kernel $(t=2)$

\begin{tabular}{ccccccc}
\hline Redundancy(percentage) & \multicolumn{2}{c}{ Amino acid composition (AAC) } & \multicolumn{2}{c}{ Dipeptide composition (DC) } & \multicolumn{2}{c}{ Hybrid (AAC+DC) } \\
\hline & Accuracy (\%) & MCC & Accuracy (\%) & MCC & Accuracy (\%) & MCC \\
\hline 30 & 92.30 & 0.84 & 93.72 & 0.87 & 93.84 & 0.88 \\
50 & 91.62 & 0.83 & 94.12 & 0.88 & 94.49 & 0.89 \\
90 & 92.44 & 0.85 & 94.67 & 0.89 & 94.86 & 0.90 \\
\hline
\end{tabular}

Table 6 Validation (accuracy) percentage by SVM models trained on AAC, DC and hybrid features.

\begin{tabular}{|c|c|c|c|c|c|c|}
\hline AAC & $\begin{array}{c}\text { Serratia marcescens } \\
\text { (127) }\end{array}$ & $\begin{array}{c}\text { Acidovorax citrulli } \\
\text { (314) }\end{array}$ & $\begin{array}{l}\text { Rhizobium fredii } \\
\text { (16) }\end{array}$ & $\begin{array}{c}\text { Solanum lycopersicum } \\
(413)\end{array}$ & $\begin{array}{c}\text { Phaseolus vulgaris } \\
\text { (159) }\end{array}$ & $\begin{array}{c}\text { Cucurbita pepo } \\
\text { (15) }\end{array}$ \\
\hline 30 & 78.74 & 98.09 & 75 & 93.46 & 94.97 & 100 \\
\hline 50 & 70.87 & 96.18 & 75 & 94.19 & 96.23 & 100 \\
\hline 90 & 70.87 & 97.77 & 75 & 93.46 & 96.86 & 100 \\
\hline \multicolumn{7}{|l|}{$D C$} \\
\hline 30 & 73.23 & 96.82 & 75 & 94.19 & 97.48 & 100 \\
\hline 50 & 75.59 & 97.45 & 68.75 & 94.92 & 96.84 & 100 \\
\hline 90 & 74.8 & 97.77 & 62.5 & 95.16 & 98.72 & 100 \\
\hline \multicolumn{7}{|c|}{$\begin{array}{l}\text { Hybrid (AAC } \\
+D C)\end{array}$} \\
\hline 30 & 74.8 & 96.5 & 75 & 95.4 & 98.74 & 100 \\
\hline 50 & 79.53 & 99.04 & 81.25 & 93.46 & 97.48 & 100 \\
\hline 90 & 81.1 & 99.04 & 79.53 & 93.95 & 98.11 & 100 \\
\hline
\end{tabular}

The accuracy is calculated by dividing the number of correct predictions by total number of protein inputs. The numbers of reviewed proteins are shown in parentheses.

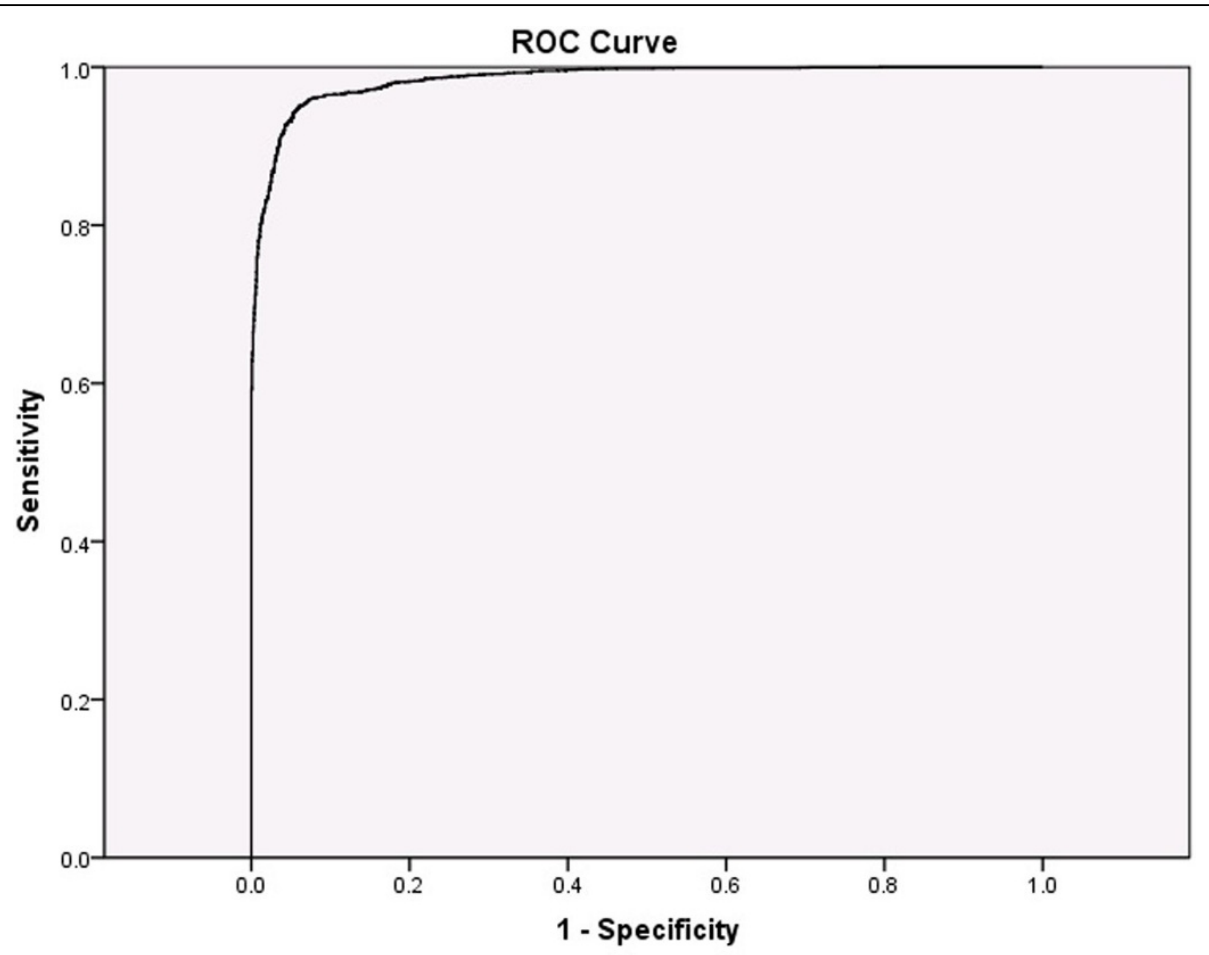

Figure 5 The ROC curve (Relative Operating Characteristic) and the area under curve for the best hybrid model at $90 \%$ redundancy. 
determine the kinds of bacterial pathogens present in food samples thus improving food security. Human pathogens that are proteobacterial in nature also exist. Specific SVM models can be trained or designed to distinguish them. Thus SVMs hold greater potential for solving a variety of problems in biology.

\section{List of abbreviations used}

SVM: Support Vector Machine; AAC: Amino Acid Composition; DC: Dipeptide Composition; FAO: Food and Agricultural Organization; ROC: Receiver Operating Characteristic; AUC: Area Under The Curve; TP: True Positive; TN: True Negative; FN: False Negative; FP: False Positive.

\section{Acknowledgements}

The authors acknowledge the support from USDA-NIFA grant number 201085605-20542, and the Oklahoma Agricultural Experiment Station whose Director has approved the manuscript for publication. The authors thank William Schneider and Jacqueline Fletcher for helpful discussion. We also thank James Borrone and Rakesh Kaundal for reading of a draft manuscript. This article has been published as part of BMC Bioinformatics Volume 13 Supplement 15, 2012: Proceedings of the Ninth Annual MCBIOS Conference. Dealing with the Omics Data Deluge. The full contents of the supplement are available online at http://www.biomedcentral.com/bmcbioinformatics/ supplements/13/S15

\section{Authors' contributions}

RV designed the study and performed the machine learning analysis and drafted the manuscript. UM conceived of the study, and participated in its design and coordination and helped to draft the manuscript. All authors read and approved the final manuscript.

\section{Competing interests}

The authors declare that they have no competing interests.

Published: 11 September 2012

\section{References}

1. Strange RN, Scott PR: Plant disease: a threat to global food security. Annual review of phytopathology 2005, 43:83-116.

2. Emerson D, Rentz JA, Lilburn TG, Davis RE, Aldrich H, Chan C, Moyer CL: A novel lineage of proteobacteria involved in formation of marine Feoxidizing microbial mat communities. PloS one 2007, 2(7):e667.

3. Melcher U, Grover V: Genomic approaches to discovery of viral species diversity of non-cultivated plants. In Recent Advances in Plant Virology. Norfolk UK: Caister Academic Press;Caranta C, Aranda MA, Tepfer M, LópezMoya JJ 2011:321-342.

4. Fletcher J, Bender C, Budowle B, Cobb WT, Gold SE, Ishimaru CA, Luster D, Melcher U, Murch R, Scherm H, et al: Plant Pathogen Forensics: Capabilities, Needs and Recommendations. MMBR 2006, 70(2):450-471.

5. Altschul SF, Gish W, Miller W, Myers EW, Lipman DJ: Basic local alignment search tool. Journal of molecular biology 1990, 215(3):403-410.

6. Verma R, Tiwari A, Kaur S, Varshney GC, Raghava GP: Identification of proteins secreted by malaria parasite into erythrocyte using SVM and PSSM profiles. BMC bioinformatics 2008, 9:201.

7. Kaundal R, Raghava GP: RSLpred: an integrative system for predicting subcellular localization of rice proteins combining compositional and evolutionary information. Proteomics 2009, 9(9):2324-2342.

8. Hu X, Wong KK, Young GS, Guo L, Wong ST: Support vector machine multiparametric MRI identification of pseudoprogression from tumor recurrence in patients with resected glioblastoma. Journal of magnetic resonance imaging: JMRI 2011, 33(2):296-305.

9. Choi S, Jiang Z: Cardiac sound murmurs classification with autoregressive spectral analysis and multi-support vector machine technique. Computers in biology and medicine 2010, 40(1):8-20.

10. Magnin B, Mesrob L, Kinkingnehun S, Pelegrini-lssac M, Colliot O, Sarazin M, Dubois B, Lehericy S, Benali H: Support vector machine-based classification of Alzheimer's disease from whole-brain anatomical MRI. Neuroradiology 2009, 51(2):73-83.
11. Vert JP: Support vector machine prediction of signal peptide cleavage site using a new class of kernels for strings. Pacific Symposium on Biocomputing Pacific Symposium on Biocomputing 2002, 649-660.

12. Furey TS, Cristianini N, Duffy N, Bednarski DW, Schummer M, Haussler D: Support vector machine classification and validation of cancer tissue samples using microarray expression data. Bioinformatics 2000, 16(10):906-914.

13. Dharmasaroja P, Dharmasaroja PA: Prediction of intracerebral hemorrhage following thrombolytic therapy for acute ischemic stroke using multiple artificial neural networks. Neurological research 2012, 34(2):120-128.

14. Naguib IA, Darwish HW: Support vector regression and artificial neural network models for stability indicating analysis of mebeverine hydrochloride and sulpiride mixtures in pharmaceutical preparation: a comparative study. Spectrochimica acta Part A, Molecular and biomolecular spectroscopy 2012, 86:515-526.

15. Dondoshansky IWY: BLASTCLUST - BLAST score-based single-linkage clustering 2000.

16. Joachims T: Learning to classify text using support vector machines. Boston: Kluwer Academic Publishers; 2002.

17. O'Dwyer L, Lamberton F, Bokde AL, Ewers M, Faluyi YO, Tanner C, Mazoyer B, O'Neill D, Bartley M, Collins DR, et al: Using support vector machines with multiple indices of diffusion for automated classification of mild cognitive impairment. PloS one 2012, 7(2):e32441.

18. Ansari HR, Raghava GP: Identification of conformational B-cell Epitopes in an antigen from its primary sequence. Immunome research 2010, 6:6.

19. Baldi P, Brunak S, Chauvin Y, Andersen CA, Nielsen H: Assessing the accuracy of prediction algorithms for classification: an overview. Bioinformatics 2000, 16(5):412-424

20. Verma R, Varshney GC, Raghava GP: Prediction of mitochondrial proteins of malaria parasite using split amino acid composition and PSSM profile. Amino acids 2010, 39(1):101-110.

21. Lu Q, Cui Y, Ye C, Wei C, Elston RC: Bagging optimal ROC curve method for predictive genetic tests, with an application for rheumatoid arthritis. Journal of biopharmaceutical statistics 2010, 20(2):401-414.

22. He X, Frey E: ROC, LROC, FROC, AFROC: an alphabet soup. Journal of the American College of Radiology: JACR 2009, 6(9):652-655.

23. Chappell FM, Raab GM, Wardlaw JM: When are summary ROC curves appropriate for diagnostic meta-analyses? Statistics in medicine 2009, 28(21):2653-2668.

24. Algarabel S, Pitarque A: ROC parameters in item and context recognition. Psicothema 2007, 19(1):163-170.

25. Higashida Y, Ideguchi T, Muranaka T, Tabata N, Miyajima R, Akazawa F, Ikeda H, Morimoto K, Ohki M, Toyofuku F, et al: [ROC analysis of detection of interval changes in interstitial lung diseases on digital chest radiographs using the temporal subtraction technique]. Nihon lgaku Hoshasen Gakkai zasshi Nippon acta radiologica 2004, 64(1):35-40.

26. Wiebringhaus R, John V, Muller RD, Hirche H, Voss M, Callies R: [ROC analysis of image quality in digital luminescence radiography in comparison with current film-screen systems in mammography]. Aktuelle Radiologie 1995, 5(4):263-267.

27. Daures JP: [Use of ROC curves in medical imaging]. Journal de radiologie 1991, 72(8-9):445-461.

28. Hannequin P, Liehn JC, Delisle MJ, Deltour G, Valeyre J: ROC analysis in radioimmunoassay: an application to the interpretation of thyroglobulin measurement in the follow-up of thyroid carcinoma. European journal of nuclear medicine 1987, 13(4):203-206.

29. Creelman CD, Donaldson W: ROC curves for discrimination of linear extent. Journal of experimental psychology 1968, 77(3):514-516.

30. Balakrishnan N: Handbook of the logistic distribution. New York: Dekker; 1992.

31. Zahr N, Arnaud L, Marquet P, Haroche J, Costedoat-Chalumeau N, Hulot JS, Funck-Brentano C, Piette JC, Amoura Z: Mycophenolic acid area under the curve correlates with disease activity in lupus patients treated with mycophenolate mofetil. Arthritis and rheumatism 2010, 62(7):2047-2054.

doi:10.1186/1471-2105-13-S15-S9

Cite this article as: Verma and Melcher: A Support Vector Machine based method to distinguish proteobacterial proteins from eukaryotic plant proteins. BMC Bioinformatics 2012 13(Suppl 15):S9. 